\title{
Sarcoidosis-related mortality in France: a multiple-cause-of-death analysis
}

\author{
Yvan Jamilloux ${ }^{1,8}$, Delphine Maucort-Boulch ${ }^{2,3,8}$, Sébastien Kerever ${ }^{4,5}$, \\ Mathieu Gerfaud-Valentin ${ }^{1}$, Christiane Broussolle ${ }^{1}$, Mireille Eb ${ }^{6}$, \\ Dominique Valeyre ${ }^{7,9}$ and Pascal Seve ${ }^{1,9}$
}

\begin{abstract}
Affiliations: ${ }^{1}$ Dept of Internal Medicine, Hôpital de la Croix-Rousse, Hospices Civils de Lyon, Université Claude Bernard-Lyon 1, Lyon, France. ${ }^{2}$ Service de Biostatistiques, Hospices Civils de Lyon, Université Claude Bernard-Lyon 1, Lyon, France. ${ }^{3}$ CNRS UMR5558, Laboratoire de Biométrie et Biologie Evolutive, Equipe Biostatistique-Santé, Villeurbanne, France. ${ }^{4}$ Dept of Anaesthesiology and Critical Care, Lariboisière University Hospital, AP-HP, Paris, France. ${ }^{5}$ University Denis Diderot - Paris VII, Paris, France. ${ }^{6}$ INSERM, CépiDc, Le Kremlin-Bicêtre, Paris, France. ${ }^{7}$ Assistance Publique - Hôpitaux de Paris, Hôpital Avicenne and Université Paris 13, Sorbonne Paris Cité, Bobigny, France. ${ }^{8}$ These authors contributed equally to this work. ${ }^{9}$ These authors contributed equally to this work.
\end{abstract}

Correspondence: Yvan Jamilloux, Service de Médecine Interne, Hôpital de la Croix-Rousse, 103 grande rue de la Croix-Rousse, 69004 Lyon, France. E-mail: yvanjamillouxahotmail.com

ABSTRACT We evaluated mortality rates and underlying causes of death among French decedents with sarcoidosis from 2002 to 2011.

We used data from the French Epidemiological Centre for the Medical Causes of Death to 1) calculate sarcoidosis-related mortality rates, 2) examine differences by age and gender, 3) determine underlying and nonunderlying causes of death, 4) compare with the general population (observed/expected ratios), and 5) analyse regional differences.

1662 death certificates mentioning sarcoidosis were recorded. The age-standardised mortality rate was 3.6 per million population and significantly increased over the study period. The mean age at death was 70.4 years (versus 76.2 years for the general population). The most common underlying cause of death was sarcoidosis. Sarcoidosis decedents were more likely to be males when aged $<65$ years. When sarcoidosis was the underlying cause of death, the main other mentions on death certificates were chronic respiratory and cardiovascular diseases. The overall observed/expected ratio was $>1$ for infectious disease, tuberculosis and chronic respiratory disease, and $<1$ for neoplasms. We observed a north-south gradient of agestandardised mortality ratio at the country level.

Despite the limitation of possibly capturing the more severe cases of sarcoidosis, this study may help define and prioritise preventive interventions.

@ERSpublications

Information from death certificates improves knowledge on sarcoidosis-related mortality http://ow.ly/lg1a302XxjQ

Editorial comment in: Eur Respir J 2016; 48: 1545-1548.

Received: March 032016 | Accepted after revision: Aug 032016 | First published online: Sept 222016

Conflict of interest: Disclosures can be found alongside this article at erj.ersjournals.com

Copyright OERS 2016 


\section{Introduction}

Sarcoidosis is a systemic disease of unknown cause. It is characterised by the formation of immune granulomas in various organs, mainly the lungs and lymphatics [1]. Sarcoidosis affects individuals from all racial/ethnic origins and occurs at any time of life. Its incidence is estimated at between 4.7 and 64 per 100000 population, and its prevalence varies from 1.0 to 35.5 per 100000 population per year [1-3]. Several studies that have used information from death certificates, at a national level, have reported lower survival rates for sarcoidosis patients compared with the general population [4-7]. In contrast, a recent cohort study of confirmed cases of sarcoidosis, performed between 1946 and 2013 in Olmsted County, Minnesota, USA, found no difference [8]. Sarcoidosis-related mortality is reported to be up to 7.6\% [4]. Most deaths are caused by advanced pulmonary fibrosis, cardiac disease and neurologic disease [9]. A recent study has shown that an integrated clinico-radiological system may capture a subset of cases of sarcoidosis that have an increased risk of mortality [10].

Few studies have determined sarcoidosis-related mortality rates at a national level $[6,7,11-15]$. In Japan, the age-adjusted mortality rate was estimated at 0.2 per million population in 1972 and 0.1 per million population in 1984 [11]. Strikingly, in Japan, cardiac involvement is the leading cause of death in patients with sarcoidosis (77\%). In the USA, age-adjusted mortality rates increased steadily from 1979 to 2007: for males this was from 1.3 to 3.76 deaths per million population and for females it was from 1.9 to 6.11 deaths per million population [6, 7]. In England, there was also a trend for increasing age-adjusted mortality rates between 1995 and 2008 (from 2.0 to 2.2 male deaths per million population and from 1.8 to 2.2 female deaths per million population) [16]. As all these studies used information from death certificates, such an increase does not necessarily reflect increased mortality but, rather, more complex phenomena, including the recognition of this disease and its reporting on death certificates.

We examined data from death certificates compiled by the French Epidemiological Centre for the Medical Causes of Death (CépiDc, INSERM) between 2002 and 2011. Using multiple-cause-of-death (MCOD) analysis, we calculated age-adjusted mortality rates for sarcoidosis, examined differences in mortality rates according to age and gender, recorded the underlying causes of death (UCDs) and assessed the geographic variability of mortality rates across French regions. The rationale for this study was to evaluate sarcoidosis-related mortality compared with the general population, the impact of the most prevalent sarcoidosis-related comorbidities and geographical differences at a country level.

\section{Methods}

Data source and retrieval

Since 2000, CépiDc has recorded all death certificates issued in France. These anonymous data are freely available to French researchers. French certificates consist of two parts and comply with the World Health Organization standards. Part I lists the "diseases related to the morbid process leading to death", in reverse order of causality. The last condition listed is defined as the UCD. Part II reports on "other significant conditions contributing to death, but not related to the disease or condition causing it". All diseases not listed as the UCD are considered as nonunderlying causes of death (NUCDs). All data are recorded in a database following the guidelines of the International Classification of Diseases, 10th Revision. For the study period, we obtained data from all death certificates that mentioned "sarcoidosis" either as the UCD or a NUCD. Only anonymous data from decedents aged $\geqslant 18$ years were analysed.

\section{MCOD analysis}

The number of death certificates that listed sarcoidosis as the UCD and the number of death certificates that listed sarcoidosis in general, whatever its place on the death certificate, were considered in the MCOD analysis. Gender and age at time of death were extracted. Direct age standardisation with the 2006 general French population, as a reference, was applied to calculate mortality rates, and estimated by year and for the study period.

For certificates that listed sarcoidosis as NUCD, the UCDs were investigated and the observed/expected ratios were calculated (adjusted for age and gender). For each UCD, the observed/expected ratio was calculated based on the proportional mortality rate for the same UCD within the general French population, between 2002 and 2011. The age-standardised mortality rates were also determined for each region in France.

\section{Statistical analysis}

Mortality rates were categorised according to age group for the entire study period. Categorical variables are presented as absolute values (with percentages), whereas continuous variables are shown as mean \pm SD. The numbers of deaths were compared according to gender, age and the cause of death (UCD or NUCD) using the Chi-squared or Fisher exact test. Proportions of sarcoidosis listed either as the UCD or a NUCD were studied over time; linear trend being tested for time. Linear regression and t-tests were used to 
compare the mean age at death by year and gender, respectively. Poisson's regressions were used to compare mortality rates and standardised mortality rates overall, by gender and age range. Differences were considered statistically significant when $\mathrm{p}<0.05$. R software version 3.0.2 (www.r-project.org/ foundation/) was used for the statistical analyses.

\section{Results}

Characteristics of decedents with sarcoidosis

Between 2002 and 2011, 5289090 adults died in France. The CépiDc recorded 1662 death certificates containing a diagnosis of sarcoidosis (mean (range) number of deaths annually 166 (132-209)). The proportion of in-hospital deaths was higher for sarcoidosis decedents than in the general population $(66.5 \%$ versus $49 \%$ ). The ratio (range) of females to males was $1.3(0.9-1.8)$ and remained stable throughout the study period. The mean \pm SD age at death was $70.4 \pm 13$ years, without a difference between females and males $(71.2 \pm 13$ and $69.2 \pm 13$, respectively). $53 \%$ of all sarcoidosis-related deaths occurred when aged $60-79$ years. Over the same period, the mean \pm SD age at death of the general population was $76.2 \pm 16$ years (figure la).

Compared with the general population, sarcoidosis-related death before the age of 65 years was statistically more frequent among males $(\mathrm{p}=0.002)$ and was statistically more frequent for females aged $\geqslant 65$ years $(\mathrm{p}<0.001)$ (figure $1 b)$. Throughout the study period, the mean age at death was significantly increased for females $(p=0.001)$, but not for males $(p=0.46$; figure $1 c)$. For females, this increase significantly differed from that recorded in the general population $(\mathrm{p}=0.007)$.

The overall age-standardised mortality rate among sarcoidosis decedents was 3.6 per million population (table 1). Between 2002 and 2011, this mortality rate increased significantly ( $p=0.006$; figure $1 \mathrm{~d}$ ).
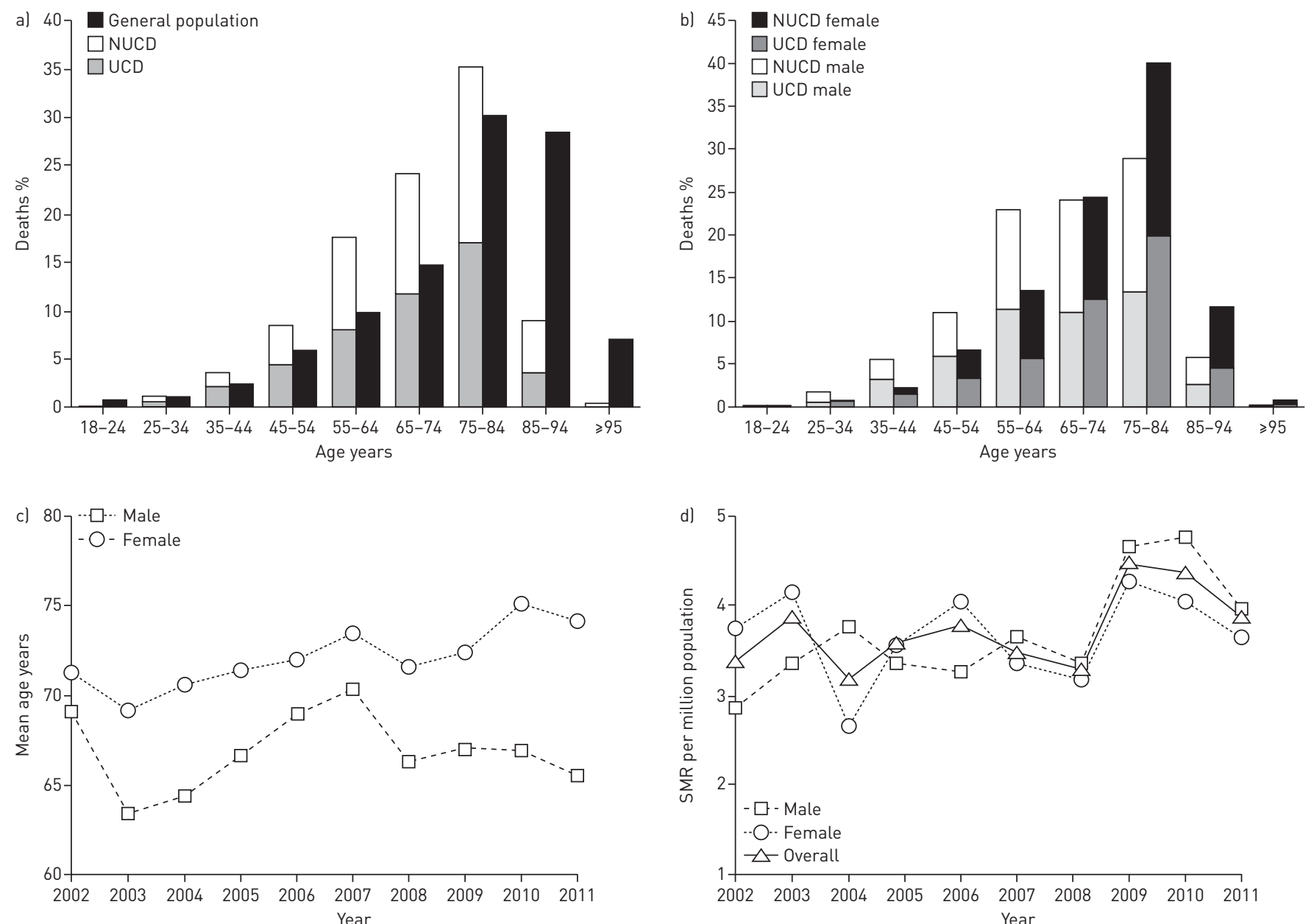

FIGURE 1 Deaths associated with sarcoidosis in France between 2002 and 2011. a) Comparison of the percentages of deaths that were sarcoidosis-related compared with the general population, according to age. UCD: underlying cause of death; NUCD: nonunderlying cause of death. b) Percentages of deaths that were sarcoidosis-related according to gender and age. c) Evolution of the mean age at death over the study period according to gender. d) Age-standardised mortality rate (SMR) stratified by gender. 
TABLE 1 Absolute number of sarcoidosis-related deaths in France between 2002 and 2011, stratified by year

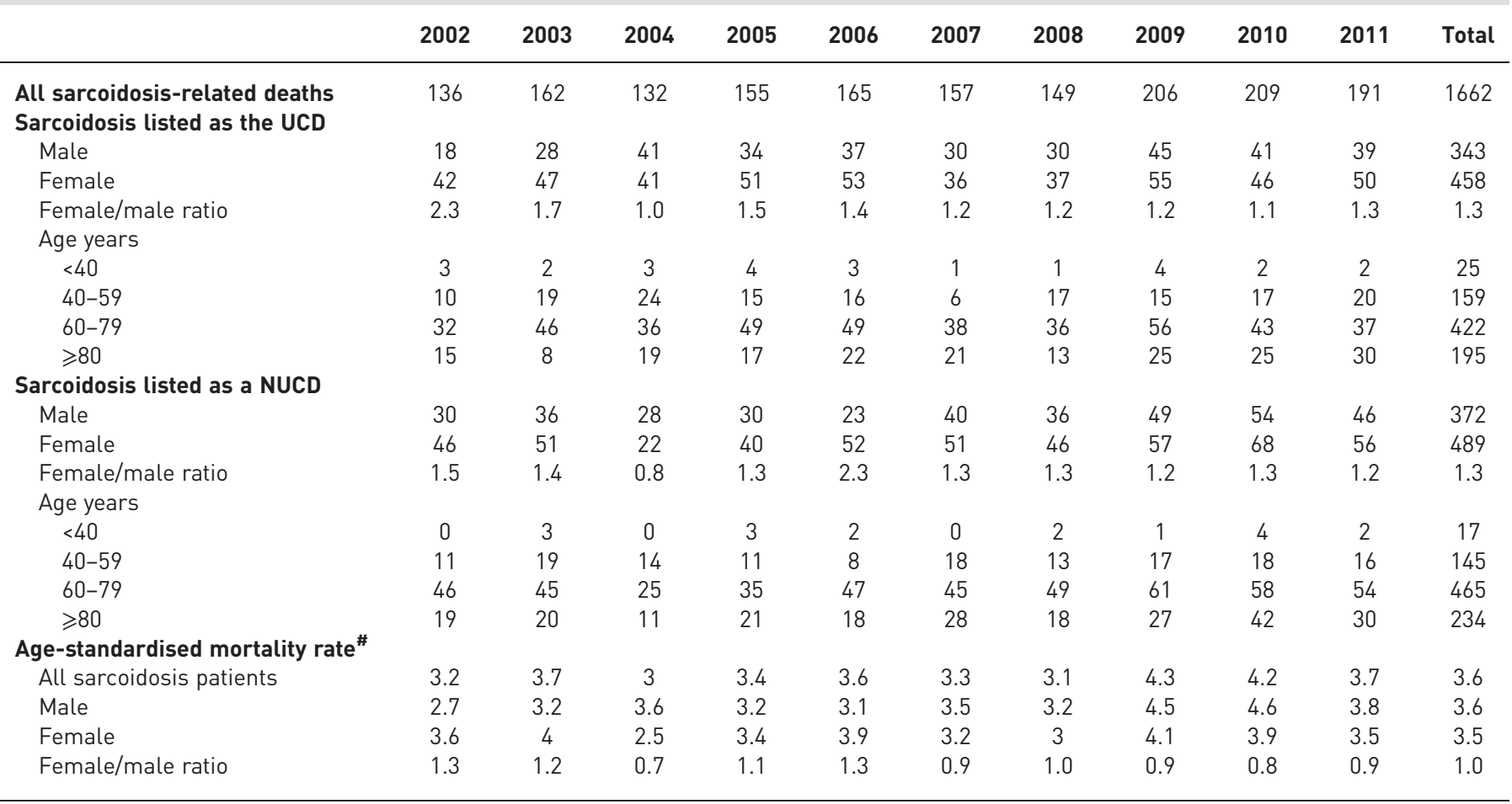

Data are presented as n, unless otherwise stated. UCD: underlying cause of death; NUCD: nonunderlying cause of death. ${ }^{\#}$ : per million population.

\section{MCOD analysis}

Sarcoidosis was listed as the UCD in 801 death certificates (48\%) and as a NUCD in 861 death certificates. The UCD/NUCD proportion was the same between females and males. There was no statistical difference between UCD and NUCD notification according to gender or age. There was no difference regarding gender ratio, age at death, in-hospital mortality or mortality rates between "sarcoidosis as the UCD" and "sarcoidosis as a NUCD" groups. Nevertheless, sarcoidosis notification as the UCD progressively decreased with age (59\% in individuals aged <40 years versus $45 \%$ in individuals aged $\geqslant 80$ years), whereas notification as a NUCD increased with age.

When sarcoidosis was reported as the UCD, the main associated causes were chronic respiratory disease, cardiovascular disease and infectious disease, with these causes being nonexclusive (i.e. one or more of these causes could be reported concomitantly as part of the process leading to death; table 2). Among the death certificates that listed chronic respiratory disease as a NUCD, the most frequent was chronic respiratory insufficiency, followed by lung fibrosis and pulmonary arterial hypertension. Among the 400 cardiovascular NUCDs, chronic heart disease was the most prevalent, followed by ischaemic heart disease and pulmonary embolism.

When sarcoidosis was listed as a NUCD, the main UCDs were malignant neoplasm, chronic disease, cardiovascular disease and infectious disease (table 3). Neoplasms were mostly solid malignant neoplasms. Among them, lung cancer was reported more often in males than females (32.5\% versus 14.1\%), mostly after the age of 50 years. Non-Hodgkin's lymphomas were the main haematologic malignancies reported as the UCD, mostly in females aged $>50$ years. Among the cardiovascular diseases, ischaemic heart disease was more often reported as the UCD in males than females (52.3\% versus $28.8 \%$ ). Among respiratory diseases, pulmonary arterial hypertension was more often reported as the UCD in females, whereas chronic respiratory insufficiency was more often reported as the UCD in males. Autoimmune diseases were reported eight times more frequently as the UCD in females than in males.

\section{Comparison of individuals with sarcoidosis and the general population}

An age- and gender-adjusted observed/expected ratio of $>1$ in sarcoidosis-associated mortality compared with the general population mortality was observed among males and females whose UCD was infectious disease (1.68), tuberculosis (12.86) or chronic respiratory disease (2.34) (tables 4 and 5). A positive association was observed between sarcoidosis and infectious disease or tuberculosis in death occurring 
TABLE 2 Nonunderlying cause of death when sarcoidosis was listed as the underlying cause of death, for the period 2002-2011

\section{Chronic respiratory disease}

Chronic respiratory insufficiency

491 (20.3)

Lung fibrosis

$306(12.7)$

Pulmonary arterial hypertension

$153(6.3)$

$32(1.3)$

$400(16.7)$

Chronic heart disease

$156(6.5)$

Ischaemic heart disease

$48(2.0)$

Pulmonary embolism

$23(1.0)$

Other

$173(7.2)$

Infectious disease

$265(11.0)$

Pneumonia or sepsis

$188(7.8)$

Tuberculosis

$13(0.5)$

Aspergillosis

$28(1.2)$

Other infections

$36(1.5)$

$104(4.3)$

73 (3.0)

Renal disease

$24(1.0)$

Autoimmune disease

$7(0.3)$

Hepatic disease

$44(1.8)$

Neoplasm

$10(0.4)$

Haematologic malignancy

5 (0.2)

Lung cancer

$29(1.2)$

Other solid malignant neoplasm

$1113(46)$

$2417(100)$

Data are presented as $\mathrm{n}(\%)$.

TABLE 3 Underlying cause of death when sarcoidosis was listed as a nonunderlying cause of death, for the period 2002-2011

\begin{tabular}{lccc} 
& Male & Female & Total \\
\hline Subjects & 372 & 489 & 861 \\
Cardiovascular disease & $65(17.5)$ & $80(16.4)$ & $145(16.8)$ \\
Pulmonary embolism & $4(1.1)$ & $6(1.2)$ & $10(1.2)$ \\
Cerebrovascular disease & $10(2.7)$ & $13(2.7)$ & $23(2.7)$ \\
Ischaemic heart disease & $34(9.1)$ & $23(4.7)$ & $57(6.6)$ \\
Other & $17(4.6)$ & $38(7.8)$ & $55(6.4)$ \\
Chronic disease & $78(21.0)$ & $86(17.6)$ & $164(19.0)$ \\
Respiratory disease & & & \\
$\quad$ Lung fibrosis & $11(3.0)$ & $12(2.5)$ & $23(2.7)$ \\
$\quad$ Pulmonary arterial hypertension & $3(0.8)$ & $10(2.0)$ & $13(1.5)$ \\
Chronic respiratory insufficiency & $18(4.8)$ & $10(2.0)$ & $28(3.3)$ \\
Autoimmune disease & $1(0.3)$ & $12(2.5)$ & $13(1.5)$ \\
Heart disease & $30(8.1)$ & $24(4.9)$ & $54(6.3)$ \\
Hepatic disease & $11(3.0)$ & $8(1.6)$ & $19(2.2)$ \\
Renal disease & $4(1.1)$ & $10(2.0)$ & $14(1.6)$ \\
Infectious disease & $32(8.6)$ & $21(4.3)$ & $89(10.3)$ \\
Pneumonia or sepsis & $16(4.3)$ & $10(2.0)$ & $37(4.3)$ \\
Tuberculosis & $6(1.6)$ & 0 & $16(1.9)$ \\
Aspergillosis & $2(0.5)$ & $26(5.3)$ & $2(0.2)$ \\
Other infections & $8(2.2)$ & $116(23.7)$ & $34(3.9)$ \\
Neoplasm & $97(26.1)$ & $24(4.9)$ & $213(24.7)$ \\
Haematologic malignancy & $17(4.6)$ & $13(2.7)$ & $41(4.8)$ \\
Lung cancer & $26(7.0)$ & $79(16.2)$ & $39(4.5)$ \\
Other solid malignant neoplasm & $54(14.5)$ & $150(30.7)$ & $133(15.4)$ \\
Other & $100(26.9)$ & & $250(29.0)$ \\
\hline
\end{tabular}

Data are presented as $\mathrm{n}$ or $\mathrm{n}(\%)$. 
TABLE 4 Age- and gender-adjusted observed/expected ratios for the underlying cause of death (UCD) when sarcoidosis was a nonunderlying cause of death (NUCD) in males ${ }^{\#}$

\begin{tabular}{|c|c|c|c|c|c|c|}
\hline & \multicolumn{2}{|c|}{ 45-64 years } & \multicolumn{2}{|c|}{$65-84$ years } & \multicolumn{2}{|c|}{$\geqslant 85$ years } \\
\hline & $\begin{array}{l}\text { Observed/expected } \\
\text { ratio }(95 \% \mathrm{CI})\end{array}$ & p-value & $\begin{array}{l}\text { Observed/expected } \\
\text { ratio }(95 \% \mathrm{CI})\end{array}$ & p-value & $\begin{array}{l}\text { Observed/expected } \\
\text { ratio }(95 \% \mathrm{CI})\end{array}$ & p-value \\
\hline Tuberculosis & $24.64(6.15-98.8)$ & $<0.001^{\Uparrow}$ & $9.46(3.05-29.36)$ & $<0.001^{\pi}$ & $24.64(6.15-98.8)$ & $<0.001^{\pi}$ \\
\hline Pneumonia & $3.23(0.81-12.91)$ & 0.098 & $0.62(0.16-2.49)$ & 0.503 & $3.23(0.81-12.91)$ & 0.098 \\
\hline Other & $2(0.64-6.19)$ & 0.232 & $1.87(0.84-4.16)$ & 0.126 & $2(0.64-6.19)$ & 0.232 \\
\hline Cerebrovascular disease & $1.66(0.62-4.41)$ & 0.313 & $0.62(0.3-1.3)$ & 0.207 & $1.66(0.62-4.41)$ & 0.313 \\
\hline Ischaemic heart disease & $0.99(0.44-2.2)$ & 0.977 & $1.04(0.65-1.65)$ & 0.87 & $0.99(0.44-2.2)$ & 0.977 \\
\hline Other & $2.82(1.75-4.53)$ & $<0.001^{\uparrow}$ & $1.14(0.78-1.66)$ & 0.497 & $2.82(1.75-4.53)$ & $<0.001^{\pi}$ \\
\hline Neoplasm & $0.47(0.3-0.74)$ & $0.001^{\pi}$ & $0.85(0.66-1.09)$ & 0.198 & $0.47(0.3-0.74)$ & $0.001^{\pi}$ \\
\hline Haematologic malignancy & $1.58(0.51-4.9)$ & 0.428 & $1.02(0.46-2.26)$ & 0.97 & $1.58(0.51-4.9)$ & 0.428 \\
\hline Lung cancer & $0.29(0.11-0.77)$ & $0.013^{9}$ & $1.08(0.69-1.69)$ & 0.752 & $0.29(0.11-0.77)$ & $0.013^{\text {शी }}$ \\
\hline
\end{tabular}

\#: for each death certificate that listed sarcoidosis as a NUCD during the period 2002-2011, the UCDs were investigated and the observed/ expected ratio was calculated; ๆ: statistically significant at $p<0.05$.

before 40 years, and tended to decrease with age. Similarly, the observed/expected ratios corresponding to chronic respiratory disease and cardiovascular disease were higher in younger deceased individuals, and decreased with age. Overall, the observed/expected ratio of pulmonary arterial hypertension was 18.88 $(\mathrm{p}<0.001)$ and the observed/expected ratio of pulmonary fibrosis was $12.35(\mathrm{p}<0.001)$. The observed/ expected ratio corresponding to cardiovascular disease was $>1$ among males and females only when aged 45-64 years (1.86 and 2.07, respectively).

Strikingly, the observed/expected ratio corresponding to neoplasm was $<1$ in young decedents (45-64 years old). The observed/expected ratio corresponding to lung cancer was $<1$ in males aged $45-64$ years, whereas it was $>3$ in females aged $\geqslant 85$ years. Moreover, for both genders, the observed/expected was $>3$ for haematologic malignancy in patients aged $\geqslant 85$ years.

TABLE 5 Age- and gender-adjusted observed/expected ratios for the underlying cause of death (UCD) when sarcoidosis was a nonunderlying cause of death (NUCD) in females ${ }^{\#}$

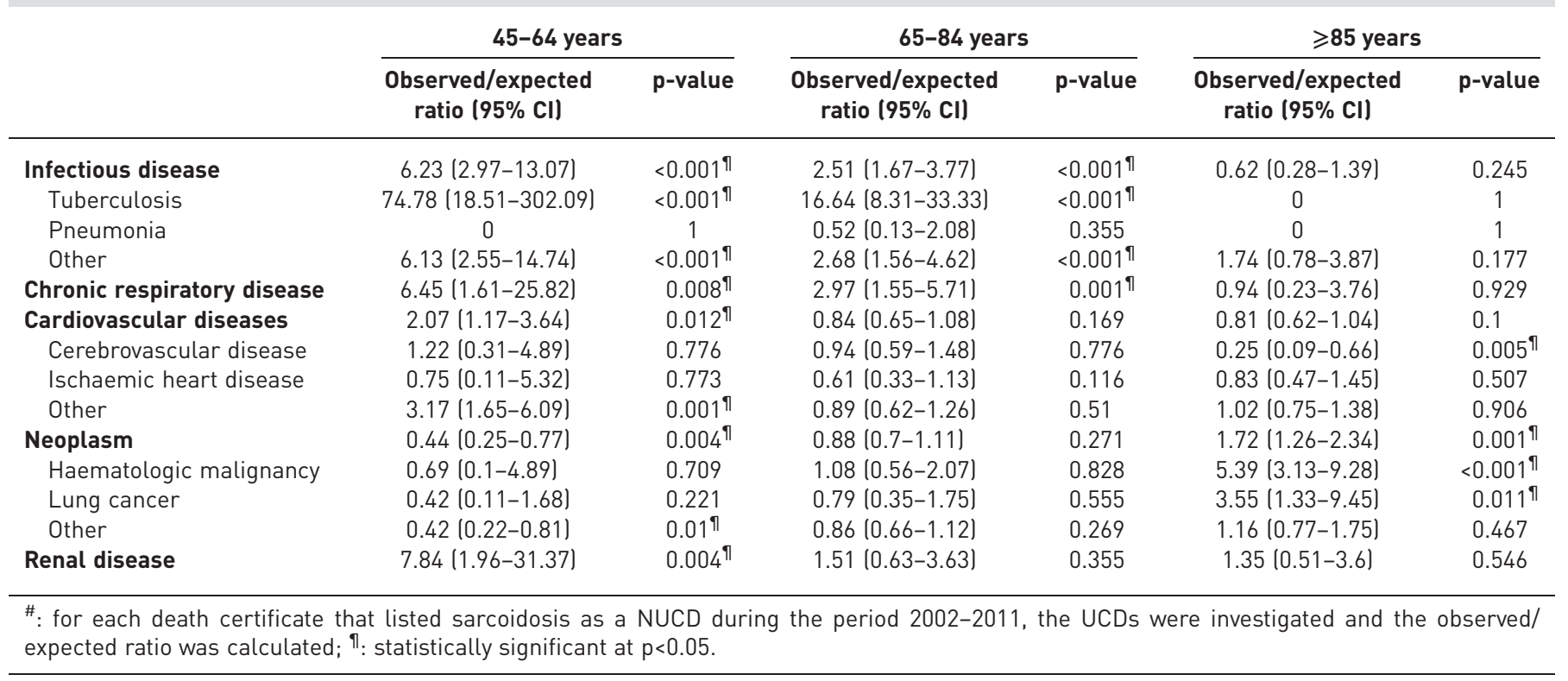




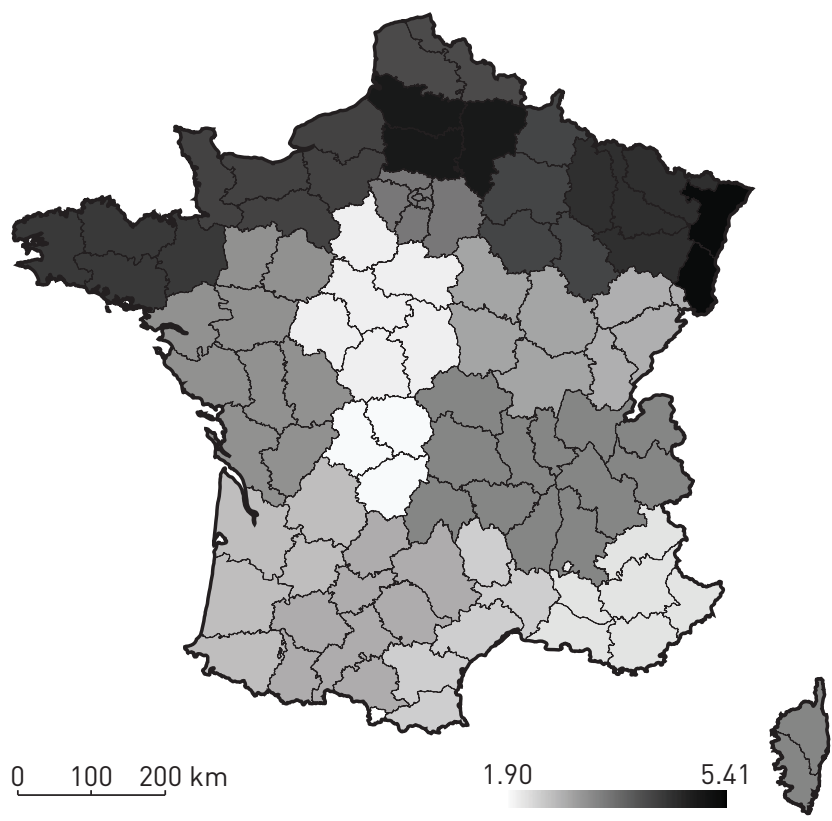

FIGURE 2 Age-standardised mortality rates (per million population) for different French regions, for the period 2002-2011.

None of the observed/expected ratios from any cause of death of individuals aged $<45$ years was statistically significant (data not shown).

\section{Geographic differences in sarcoidosis mortality}

Age-standardised mortality rates for different French geographic regions were calculated using MCOD analysis (figure 2). Mortality rates ranged from 1.98 to 5.40 per million population, with northern regions having higher mortality rates. Data from French overseas territories were not available.

\section{Discussion}

For this study, we took advantage of the exhaustive recording of all French death certificates by CépiDc since 2002 and performed the largest MCOD analysis in a European country. Mainly, we found that: 1) the mean age at death reported on death certificates that listed sarcoidosis was lower than for the general population over the same time period, by about 6 years, 2) the UCD was sarcoidosis in half of the cases, 3) before the age of 65 years deaths were more frequent in males, 4) there was a significant increase in standardised mortality rates over the study period and 5) sarcoidosis-related mortality was higher in northern regions.

Contrary to cohort studies that are often performed in restrained numbers of medical facilities and for limited periods of time, MCOD analyses provide comprehensive data at a country level with complete coverage of all deaths over selected periods of time, thus avoiding selection bias [17].

However, the MCOD analyses have some limitations: indeed, there is no way to assess the accuracy of diagnoses reported in the dataset (including histological confirmation). For example, sarcoidosis may not have been declared on the death certificates of some patients with a past clinically occult disease and/or spontaneous remission. More symptomatic, long-standing and severe phenotypes may be over-represented, leading to overestimation of sarcoidosis-related mortality. Thus, such findings may not be relevant to the whole spectrum of patients with sarcoidosis.

Moreover, in such studies, it is not possible to exclude that some patients with a alternative diagnosis (e.g. tuberculosis or sarcoid-like reactions) may have been over-reported to have sarcoidosis.

Some certifiers may also have appraised sarcoidosis complications, such as pulmonary hypertension or fibrosis, as the UCD, whereas others may have considered that they were NUCDs. Thus, mortality rates must not be considered as surrogates for the incidence of this disease. This means that MCOD analysis is not performed to provide information about fatality, but rather crude mortality rates [18-20].

Another limitation of our study is a lack of data on ethnicity, socioeconomic status and environment. We did not study the links between occupational exposure and sarcoidosis-related death. However, a recent paper has reported a greater odds ratio for cases where there was occupational exposure and a difference between genders regarding some specific exposures [21]. 
In accordance with our results, SwIGRIS et al. [4] reported a mean \pm SD age of death of $57.2 \pm 1.8$ years in decedents with sarcoidosis compared with $71.4 \pm 1.1$ years for the nonsarcoidosis population in the USA over the period 1988-2007. Although it has been stated that sarcoidosis patients have lower survival rates than the general population, a confirmed increased risk of death in the whole spectrum of sarcoidosis patients can only be identified in prospective studies, which are very difficult to conduct on a large scale. UNGPRASERT et al. [8] recently reported on a prospective cohort study, and found no difference in mortality rates between sarcoidosis patients and the general population. The differences seen in the MCOD analyses (ours and SwigRIs et al.'s [4]) probably reflect the differences between a subgroup of patients with sarcoidosis severe enough to be notified on a death certificate and the general population.

Interestingly, as compared with the general population, the majority of decedents aged $<65$ years were males, whereas females were the majority in those who died when aged $\geqslant 65$ years. These observations are in accordance with previous epidemiological reports of two incidence peaks in sarcoidosis: the first being observed in young adults (mainly males exhibiting systemic features) and the second observed in older patients (more likely to be Caucasian females with ocular sarcoidosis) [15, 16]. Our data agree with previous reports conducted in the USA and England, showing an increased female/male mortality ratio in older age strata $[4,5,13]$. Finally, we found an increased age at death over the study period that was significant only for females with sarcoidosis. Hypotheses include better management of sarcoidosis or related comorbidities through optimised monitoring, the use of novel therapeutics and better prevention of side-effects.

Over the study period, we observed a significant increase in age-standardised mortality rates. This finding has also been reported in previous studies $[4,5,12,13,22]$. Nevertheless, this increase seems to be restricted to Caucasian ethnicity [12]. Various hypotheses have been formulated, such as an increased incidence or increased severity of sarcoidosis, but the most likely reason may be improved recognition of this disease by physicians and the use of chest computed tomography in difficult cases.

Overall, sarcoidosis was mentioned as the UCD in $48 \%$ of death certificates. This rate remained stable throughout the study period, but tended to decline with increasing age, whereas sarcoidosis notification as a NUCD increased with age. We speculate that this could be caused by 1) a decreased severity of sarcoidosis in the elderly [23] or 2) the accumulation of comorbidities and/or complications in the elderly [16]. Such variations have been frequently reported in other MCOD analyses [24].

When sarcoidosis was the UCD, the most common NUCDs were chronic respiratory insufficiency, lung fibrosis and pulmonary hypertension. In previous reports, lung fibrosis was reported as a contributing factor in $9 \%$ of deaths, but may have been under-reported according to the authors [4]. Pulmonary hypertension was mentioned on $2.8-7.1 \%$ of death certificates of sarcoidosis decedents [4]. Considering that they are known complications of sarcoidosis (mainly stage 4 patients) [7], the number of patients dying from lung fibrosis or pulmonary hypertension should be interpreted carefully. Indeed, death certifiers may simply have written "sarcoidosis" on death certificates without mentioning lung fibrosis or pulmonary hypertension. Moreover, some certifiers may have considered these complications as the UCD, whereas other may have mentioned them as NUCDs [25]. Finally, this observation is likely to reflect a subgroup of patients with more cases of chronic or severe sarcoidosis and who have a greater risk of dying from sarcoidosis.

Cardiovascular aetiology was described as a NUCD in 17\% of decedents with sarcoidosis as the UCD. This is consistent with the $25 \%$ rate reported in the USA [4]. Such a rate suggests that, at the time of death, sarcoidosis was severe enough to warrant coding by death certifiers. In line with our results, a recent publication has associated sarcoidosis with pulmonary embolism and congestive heart failure in patients admitted into UK hospitals over a period of 35 years [26]. In our study, it was not possible to distinguish between nonspecific and sarcoidosis-specific heart disease.

When sarcoidosis was mentioned as a NUCD, neoplasms were the main UCD followed by cardiovascular disease and infection. Neoplasms were reported more often in decedents aged $>50$ years; lung cancer being predominant in males, whereas females were more likely to have non-Hodgkin's lymphoma reported as the UCD. Importantly, observed/expected ratios, which quantify the increase or decrease in mortality due to sarcoidosis, as compared with the general population, were found to be $>1$ for only infectious diseases (including tuberculosis) and chronic respiratory disease. Although the observed/expected ratio was high for tuberculosis, the raw number of deaths was very low (29 cases). As stated above, there was also uncertainty about a diagnosis of tuberculosis due to the nature of the data. Thus, in some cases, sarcoidosis and tuberculosis may have been misdiagnosed.

A cardiovascular-associated increase in mortality was only significant for decedents aged 45-64 years. In contrast, haematologic malignancy was more likely to lead to death in decedents aged $\geqslant 85$ years.

Strikingly, the overall observed/expected ratio was $<1$ among sarcoidosis decedents whose death certificates indicated a neoplasm. Further analyses by age and gender revealed that this was only true and significant 
for those aged between 45 and 65 years at death. Solid neoplasms other than lung cancer were significantly lower in sarcoidosis patients aged 45-64 years and lung cancer was only significantly lower in males aged 45-64 years. One possible explanation may be a lower number of smokers within sarcoidosis patients, as this was reported previously $[27,28]$. Another explanation may be a lower probability of reporting sarcoidosis when a neoplasm was the UCD. In contrast, mortality caused by haematologic malignancy was higher in older individuals (aged $\geqslant 85$ years) and mortality due to malignancy, either haematologic or lung cancer, was higher in females aged $\geqslant 85$ years. In a previous study in the UK, LE JEUnE et al. [29] reported an overall increased incidence of cancer in sarcoidosis, but this was mostly due to increased incidence of skin cancers. For pneumonia and cerebrovascular diseases, observed/expected ratios $<1$ were observed in females and in those aged $\geqslant 85$ years, respectively. This may reflect better medical management (including smoking cessation counselling) and earlier diagnoses rather than protection due to sarcoidosis itself. In the USA, MisRaeidi et al. [12] reported less deaths caused by pneumonia in Caucasian females with sarcoidosis than in a control group comprised of patients with rheumatoid arthritis. They also found that Caucasians in the sarcoidosis group were less likely to have diabetes or a neoplasm, but were more likely to have heart failure. Nevertheless, their study was designed to analyse racial differences and the comparison was made using an identified control group rather than the general population, thus precluding a formal comparison. In France, ethnic statistics are forbidden; thus, we were unable to compare patients from different ethnicities. Similarly, data on residency were not available.

Finally, we did find a north-south gradient of age-standardised mortality at the country level. Despite the lack of data on incidence and prevalence of sarcoidosis in France, this gradient is more likely to reflect the prevalence gradient constantly reported at the European continent level [15, 30-32]. Such a difference is hypothesised to be due to environmental or occupational exposures rather than disparities in healthcare management.

\section{Conclusions}

Due to intrinsic limitations, our study is not applicable to the whole spectrum of patients with sarcoidosis and may overestimate sarcoidosis-related mortality. Nevertheless, considering data from death certificates, sarcoidosis-related mortality rates increased in France over the period 2002-2011. Sarcoidosis was the UCD in half of the cases. The increased mortality associated with sarcoidosis affected males at a younger age, whereas it affected older females. Finally, there is a clear geographic impact with sarcoidosis-related mortality being higher in northern regions. Improving our knowledge on sarcoidosis-related mortality may help define and prioritise preventive interventions.

\section{References}

Valeyre D, Prasse A, Nunes H, et al. Sarcoidosis. Lancet 2014; 383: 1155-1167.

Iannuzzi MC, Fontana JR. Sarcoidosis: clinical presentation, immunopathogenesis, and therapeutics. JAMA 2011; 305: 391-399.

3 Baughman RP, Lower EE, du Bois RM. Sarcoidosis. Lancet 2003; 361: 1111-1118.

4 Swigris JJ, Olson AL, Huie TJ, et al. Sarcoidosis-related mortality in the United States from 1988 to 2007. Am J Respir Crit Care Med 2011; 183: 1524-1530.

5 Gideon NM, Mannino DM. Sarcoidosis mortality in the United States 1979-1991: an analysis of multiple-cause mortality data. Am J Med 1996; 100: 423-427.

6 Gribbin J, Hubbard RB, Le Jeune I, et al. Incidence and mortality of idiopathic pulmonary fibrosis and sarcoidosis in the UK. Thorax 2006; 61: 980-985.

7 Nardi A, Brillet P-Y, Letoumelin P, et al. Stage IV sarcoidosis: comparison of survival with the general population and causes of death. Eur Respir J 2011; 38: 1368-1373.

8 Ungprasert P, Carmona EM, Utz JP, et al. Epidemiology of sarcoidosis 1946-2013: a population-based study. Mayo Clin Proc 2016; 91: 183-188.

9 Costabel U, Hunninghake GW. ATS/ERS/WASOG statement on sarcoidosis. Sarcoidosis Statement Committee. American Thoracic Society. European Respiratory Society. World Association for Sarcoidosis and Other Granulomatous Disorders. Eur Respir J 1999; 14: 735-737.

10 Walsh SL, Wells AU, Sverzellati N, et al. An integrated clinicoradiological staging system for pulmonary sarcoidosis: a case-cohort study. Lancet Respir Med 2014; 2: 123-130.

11 Yamaguchi M, Hosoda Y, Sasaki R, et al. Epidemiological study on sarcoidosis in Japan. Recent trends in incidence and prevalence rates and changes in epidemiological features. Sarcoidosis 1989; 6: 138-146.

12 Mirsaeidi M, Machado RF, Schraufnagel D, et al. Racial difference in sarcoidosis mortality in the United States. Chest 2015; 147: 438-449.

13 Duncan ME, Goldacre MJ. Mortality trends for tuberculosis and sarcoidosis in English populations, 1979-2008. Int J Tuberc Lung Dis 2012; 16: 38-42.

14 Nicholson TT, Plant BJ, Henry MT, et al. Sarcoidosis in Ireland: regional differences in prevalence and mortality from 1996-2005. Sarcoidosis Vasc Diffuse Lung Dis 2010; 27: 111-120.

15 Hillerdal G, Nöu E, Osterman K, et al. Sarcoidosis: epidemiology and prognosis. A 15-year European study. Am Rev Respir Dis 1984; 130: 29-32.

16 Morimoto T, Azuma A, Abe S, et al. Epidemiology of sarcoidosis in Japan. Eur Respir J 2008; 31: 372-379.

17 Reich JM. Mortality of intrathoracic sarcoidosis in referral $v s$ population-based settings: influence of stage, ethnicity, and corticosteroid therapy. Chest 2002; 121: 32-39.

18 Reich JM. Sarcoidosis mortality. Am J Respir Crit Care Med 2012; 185: 461-462. 
Reich JM. Mortality vs case fatality in the assessment of sarcoidosis lethality. Chest 2015; 147: e65.

Baughman RP, Lower EE. Who dies from sarcoidosis and why? Am J Respir Crit Care Med 2011; 183: 1446-1447.

Liu H, Patel D, Welch A, et al. Association between occupational exposures and sarcoidosis: an analysis from death certificates in the United States, 1988-1999. Chest 2016; 151: 289-298.

2 Hanley A, Hubbard RB, Navaratnam V. Mortality trends in asbestosis, extrinsic allergic alveolitis and sarcoidosis in England and Wales. Respir Med 2011; 105: 1373-1379.

3 Nowiński A, Puścińska E, Goljan A, et al. The influence of comorbidities on mortality in sarcoidosis: a observational prospective cohort study. Clin Respir J 2015 [in press; DOI: 10.1111/crj.12398].

Thomas G, Mancini J, Jourde-Chiche N, et al. Mortality associated with systemic lupus erythematosus in France assessed by multiple-cause-of-death analysis. Arthritis Rheumatol 2014; 66: 2503-2511.

Redelings MD, Wise M, Sorvillo F. Using multiple cause-of-death data to investigate associations and causality between conditions listed on the death certificate. Am J Epidemiol 2007; 166: 104-108.

6 Crawshaw AP, Wotton CJ, Yeates DGR, et al. Evidence for association between sarcoidosis and pulmonary embolism from 35-year record linkage study. Thorax 2011; 66: 447-448.

7 Newman LS, Rose CS, Bresnitz EA, et al. A case control etiologic study of sarcoidosis: environmental and occupational risk factors. Am J Respir Crit Care Med 2004; 170: 1324-1330.

Valeyre D, Soler P, Clerici C, et al. Smoking and pulmonary sarcoidosis: effect of cigarette smoking on prevalence, clinical manifestations, alveolitis, and evolution of the disease. Thorax 1988; 43: 516-524.

9 Le Jeune I, Gribbin J, West J, et al. The incidence of cancer in patients with idiopathic pulmonary fibrosis and sarcoidosis in the UK. Respir Med 2007; 101: 2534-2540.

Rybicki BA, Major M, Popovich J Jr, et al. Racial differences in sarcoidosis incidence: a 5-year study in a health maintenance organization. Am J Epidemiol 1997; 145: 234-241.

1 Byg K-E, Milman N, Hansen S. Sarcoidosis in Denmark 1980-1994. A registry-based incidence study comprising 5536 patients. Sarcoidosis Vasc Diffuse Lung Dis 2003; 20: 46-52.

Hosoda Y, Sasagawa S, Yasuda N, et al. Epidemiology of sarcoidosis: general approach. In: Baughman RP, ed. Sarcoidosis. Boca Raton, Taylor \& Francis, 2006; pp. 13-46. 